Aus dem pharmakologischen Institut der Universitat Leipzig.

\title{
Ueber Methylenverbindungen und einige andere Derivate der m-Dioxybenzole.
}

\author{
Von Dr. A. Luther.
}

(Eingegangen den 8. X. 1906.)

Die Methylenverbindungen der Phloroglucine und des Orcins lassen sich, wie R. Boehm') und O. Simon') gezeigt haben, durch Natronlauge und Zinkstaub in homologe Phenole zerlegen; man kann anf diesem Wege rom Phloroglucin bis zam 1.3.5.Trimethylphloro. glucin and vom Orcin bis zam 1.2.3-Trimethylphendiol (4.6) gelangen.

Es bot einiges Interesse, nach dieser Richtung anch Methylenbisresorcin and einzelne andere leichter zugängliche Derivate von Metadioxybenzolen za untersuchen.

Im Methylenbisresorcin wird das Brtickenmethylen in 0-Stellang zn je einem Hydroxyl des Resorcinmolekblls angenommen. Unter dieser - soweit aus der Literatar ersichtlich ist - noch nicht direkt bewiesenen Voraussetzung ist bei der Spaltung der Verbindung neben Resorcin Kresor cin [1-Methylphendiol (2.4.)] zo erwarten.

Es stellte sich zundchst heraus, daß Methylenbisresorcin ron Natronlange and Zinkstaub auch bei Wasserbadtemperator wenig angegriffen wird; nur durch länger fortgesetztes Kochen mit den genannten Agentien und unter reichlicher Entstehung anderweitiger Zersetzungsprodukte konnte es so zerlegt werden, da $B$ ca. $20 \%$ der angewandten Menge an homologen Phenolen gewonnen wurden.

Um etwa vorhandenes Kresorcin za identifizieren, war ex notig dieses Phenol genauer kennen zu lernen. Es ist bekanntlich zuerst von Knecht ${ }^{2}$ ) dargestellt; Dinitro., Dinitrosokresorcin- und Kresorcincarbonsăure sind von Kostane $\mathrm{cki}^{4}$ ), ein Dithioderivat von Klason ${ }^{5}$ ) beschrieben.

Bei der Darstellnng großerer Mengen von Kresorcin ging ich vom Paratoluidin ans and befolgte im wesentlichen die von

1) Annal. 329, 269.

9) Ibid. 329, 301 .

8) Annal. 215, 92.

4) Ber. 20, 3135, 18, 3203.

8) Ber. 20, 355 .

Arch. d. Pharm. CCXXXXIV, Bds, 8. Heft. 
Nölting and Collin ${ }^{1}$ ) und von Wallach$^{2}$ ) gegebenen Vorschriften; das Rohphenol wurde durch Vakuumdestillation gereinigt.

In seinem Verhalten gegen Benzoylchlorid zeigte Kresorcin die Eigentümlichkeit, daß es beim Benzoylieren in Sødalösung ein gut krystallisierendes Monobenzo at (Schmp. 115-116 ${ }^{\circ}$ ) bildet, das in Alkalilauge noch löslich ist und bei erneuter Einwirkung von Benzoylchlorid in Natronlauge in das alkaliunlösliche Dibenzoat (Schmp. $83^{\circ}$ ) tibergeht.

Diacetylkresorcin ist eine nicht erstarrende ölige Flüssigkeit. (Sdp. 293-2950.)

Bei der Einwirkung von Brom, resp. Chlor im Ueberschuß, entstehen aus Kresorcin als höchsthalogenierte Derivate Tetrabromkresorcin (Schmp. 99-100 $)$ resp. Tetrachlorkresorcin (Schmp. 69-70'), beide in Sodalösung unlöslich. Tetrachlorkresorcin ist durch Zinnchlorür nnd Salzsäure leicht zu Dichlorkresorcin (Schmp. $\left.78-79^{\circ}\right)$ zu reduzieren. Durch vorsichtiges Bromieren in Chloroform erhält man ein Dibromid (Schmp. 86-87 ${ }^{\circ}$ ).

Durch Digestion der alkoholischen Lösung mit 2//y Mol. Diazoamidobenzol entsteht Kresorcind isaz obenzol (Schmp. $211^{\circ}$ ).

In dem aus Methylenbisresorcin erhaltenen Phenolgemenge war Resorcin leicht nachzuweisen; die Identifizierung des zweiten Phenols als K resorcin [-1.Methylphendiol (2.4)] gelang nur mit Hilfe der Dinitroverbindungen beider Phenole. Nachdem von $\mathrm{Fitz}^{8}$ ) für Resorcin angegebenen Verfahren verwandelte ich das Phenolgemenge in die Dinitrosoverbindangen und oxydierte letztere in Aethersuspension durch Schütteln mit Salpetersäure vom spez. Gew. 1,3. Die beiden Nitroprodukte sind durch ibre verschiedene Löslichkeit in Wasser leicht von einander zu trennen, zuerst schied sich aus der Lبosung Dinitroresorcin (Schmp. $142^{\circ}$ ) ab, dann Dinitrokresorcin in feinen Nadeln (Schmp. 90\%). Die Produkte wurden durch die Elementaranalyse identifiziert.

Aus Kresorcin erhält man mit Formaldehyd leicht und fast quantitativ Methylenbiskresorcin (Schmp. 195-200 ${ }^{\circ}$, unter Zersetzung), meistens mikrokristallinisch, zuweilen auch in Form größerer Prismen. Bei der Spaltung mit Natronlauge und Zinkstaub zerfallt es in Xylorcin [1-3-Dimethylphendiol (4-6)] (Schmp. 124 bis $125^{\circ}$ ) and $\mathrm{Kres}$ or cin. Methylen tritt also auch bei der Kordensation von Kresorcin mit Formaldehyd in O-Stellung zu je einem Hydroxyl in beiden Molekülen.
1) Ber. 17, 263.
2) Annal. 234, 3.
8) Ber. 8, 631. 
Xylorcin warde zwecks Identifizierung nach dem Verfahren von $\mathrm{P} f \mathrm{a} \mathrm{f}^{1}$ ) synthetisch dargestellt. Neu wurde bei dieser Gelegenheit beobachtet, daß das Phenol aus wässerigen Lösungen, je nach deren Konzentration wasserfrei in derben polygonalen Platten, oder mit $1 \mathrm{Mol} . \mathrm{H}_{\mathbf{2}} \mathrm{O}$ in großen langen Prismen auskrystallisiert. Mit Eisenchlorid färbt sich die wässerige Lösung rasch vorübergehend blau.

Xylorcindibenzoat, farblose Prismen, schmilzt bei $155^{\circ}$. Bei der Einwirkung von Brom in Chloroform entstand ein Monobromderivat (Schmp. 119-120). Mit Diazoamidobenzol kuppelt Xylorcin nicht.

Das mit Hilfe von Formaldehyd in quantitativer Ausbeute erhältliche Methylenbis x y lor c in krystallisiert aus verdünntem Alkohol gut in sechsseitigen Tafeln mit 1 Mol. Krystallwasser. Wasserfrei schmilzt es bei $251^{\circ}$. Durch Natronlauge und Zinkstaub wird diese Methylenverbindung auch in der Kochhitze nicht angegriffen; ibre Konstitation läßt sich also auf diesem Wege nicht ermitteln. Einen Fingerzeig bietet aber in dieser Beziehung, daß Methylenbisxylorcin mit Diazoamidobenzol kuppelt. Das Brückenmethylen könnte in der Verbindung die Stelle zwischen zwei Kernmethylen oder zwei Hydro. xylen einnehmen; im ersteren Falle wären zwei Methine in 0-Stellung, im letzteren in $\mathrm{m}$-Stellung za Hydroxyl frei; da nach der bisherigen Erfahrung der Eintritt des Azorestes in m-Stellang zu Hydroxyl hochst unwahrscheinlich ist, wird wohl die erstere Konstitution zntreftend sein. Das bei der Spaltung dieser Verbindang theoretisch zu erwartende 1.2.3-Trimethylphendiol (4.6) ist von O. Simon (l. c.) ang Methylenbisorcin dargestellt worden. Ich konute konstatieren, $\mathrm{da} B$ es in sehr guter Ausbeute mit Formaldehyd die entsprechende Methylenverbindung liefert, daß aber auch diese von Natronlange und Zinkstaub nicht mehr angegriffen wird, somit die Darstellung von Tetramethylphendiol auf diesem Wege nicht möglich ist.

\section{Experimenteller Teil.}

\section{Trennung der Phenole nach der Spaltung des Methylenbisresorcin.}

Die anf $0^{\circ}$ abgekuhlte Lösung von $10 \mathrm{~g}$ des Phenolgemenges in $500 \mathrm{~g}$ Wasser wurde mit $12 \mathrm{~g}$ Essigsăure und hierauf einer konzentrierten Losung von $14 \mathrm{~g}$ Natriumnitrit versetzt, das Gemisch nach $1 / 4$ Stunde in $100 \mathrm{~g}$ verdünnter Schwefelsäure gegossen und der hierbei entstandene Niederschlag nach einer Stunde abfiltriert. Die Dinitrosoprodukte oxydiert man, ohne weitere vorherige Reinigung, nach dem Trocknen, indem man die fein zerriebenen, in Aether suspendierten

1) Ber. 16, 1138. 
Krystalle durch Schtitteln mit Salpetersäure (spez. Gew. 1,a) im Scheidetrichter in Lơsung bringt; den Aether wäscht man zweimal mit Wasser und läßt ihn dann abdunsten; die so erhaltenen Dinitrophenole trennt man durch fraktionierte Krystallisation aus Wasser; Dinitrokresorcin bleibt als das leichter lösliche in der Matterlauge, und krystallisiert schließlick in feinen langen Nadeln, die sich leicht von den zuerst abgeschiєdenen gelben Blättchen des Dinitroresorcins unterscheiden lassen.

Dinitrokresorcin. Schmp. $90^{\circ}$.

1. $0,1108 \mathrm{~g}$ gaben bei $20^{\circ}$ und $752 \mathrm{~mm} 13,05 \mathrm{ccm}$ Stickgas, entsprechend $0,01477 \mathrm{~N}$.

2. $0,0979 \mathrm{~g}$ gaben bei $20^{\circ}$ und $752 \mathrm{~mm} 11,57 \mathrm{ccm}$ Stickgas, entsprechend $0,01309 \mathrm{~N}$.

3. $0,1434 \mathrm{~g}$ gaben $0,2057 \mathrm{CO}_{\mathrm{g}}$ und $0,0398 \mathrm{Hg}_{\mathrm{g}} \mathrm{O}$.

\begin{tabular}{|c|c|c|c|}
\hline Berechnet für & & efunde & \\
\hline $\mathrm{C}_{7} \mathrm{H}_{6} \mathrm{~N}_{2} \mathrm{O}_{6}:$ & 1. & 2. & 3. \\
\hline $\mathrm{C} \quad 39,25$ & - & - & 39,08 \\
\hline H $\quad \mathbf{2 , 8 0}$ & - & - & 3,10 \\
\hline N 13,08 & 13,33 & 13,37 & - \\
\hline
\end{tabular}

Dinitroresorcin. Schmp. $142^{\circ}$.

1. $0,1656 \mathrm{~g}$ gaben $0,2163 \mathrm{CO}_{\mathrm{g}}$ und $0,0337 \mathrm{H}_{\mathbf{2}} \mathrm{O}$.

2. $0,1224 \mathrm{~g}$ gaben bei $16^{\circ}$ und $754 \mathrm{~mm} 18,08 \mathrm{ccm}$ Stickgas, entsprechend $0,0174 \mathrm{~N}$.

\begin{tabular}{|c|c|c|}
\hline Berechnet für & \multicolumn{2}{|c|}{ Gefunden: } \\
\hline $\mathrm{C}_{6} \mathrm{H}_{4} \mathrm{~N}_{2} \mathrm{O}_{6}:$ & 1. & 2. \\
\hline C 36,00 & 35,62 & 一 \\
\hline 2,00 & 2,29 & - \\
\hline N 14,00 & - & 14,25 \\
\hline
\end{tabular}

Dibromkresorcin (Schmp. 86-870) $\mathrm{C}_{7} \mathrm{H}_{6} \mathrm{Br}_{2} \mathrm{O}_{2}$. Ein Mol. in Chlorotorm suspendiertes Kresorcin wird successive mit $3 \mathrm{Mol}$. Brom versetz.t. Der nach dem Abdunsten verbleibende Rückstand wird durch Abpressen zwischen Fließpapier und Umkrystallisieren aus heißem Wasser gereinigt. Lange weiße Nadeln, leicht loslich in Alkohol und heißem Wasser. Ausbeute ca. $90 \%$.

1. $0,1869 \mathrm{~g}$ gaben $0,2501 \mathrm{Ag} \mathrm{Br}$, entsprechend $0,1064 \mathrm{Br}$.

2. $0,1975 \mathrm{~g}$ gaben $0,2635 \mathrm{Ag} \mathrm{Br}$, entsprechend $0,1121 \mathrm{Br}$.

3. $0,3200 \mathrm{~g}$ gaben $0,3533 \mathrm{CO}_{2}$ und $0,0680 \mathrm{H}_{8} \mathrm{O}$.

Berechnet für

\begin{tabular}{ccccc}
\multicolumn{2}{c}{$\mathrm{C}_{7} \mathrm{H}_{6} \mathrm{Br}_{2} \mathrm{O}_{8}:$} & 1. & 2. & 3. \\
$\mathrm{C}$ & 29,83 & - & - & 30,10 \\
$\mathrm{H}$ & 2,13 & - & - & 2,50 \\
$\mathrm{Br}$ & 57,09 & 56,93 & 56,76 & -
\end{tabular}


Tetrabromkresorcin (Schmp. 99-100 $)^{\circ} \mathrm{C}_{7} \mathrm{H}_{4} \mathrm{Br}_{4} \mathrm{O}_{2} . \quad 2,0 \mathrm{~g}$ Kresorcin in $20 \mathrm{~T}$. Wasser gelöst, werden nach und nach einer Mischung von $14 \mathrm{~g}$ Brom und $400 \mathrm{~g}$ Wasser unter krhiftigem Umschütteln zogegeben. Das Reaktionsprodukt abgesaugt, getrocknet, einmal ans Schwefelkohlenstoff, dann zweimal aus Ligroin umkrystallisiert. Rhombische Tafeln, leicht löslich in Alkohol, unlöslich in Sodalosang, aus Jodkaliumlösung Jod abscheidend; in Natronlauge unter Auftreten von Bromoformgeruch and Zersetzung löslich.

1. 0,2053 g gaben $0,3483 \mathrm{Ag} \mathrm{Br}$.

2. $0,2095 \mathrm{~g}$ gaben $0,3560 \mathrm{AgBr}$.

3. $0,5170 \mathrm{~g}$ gaben $0,3619 \mathrm{CO}_{2}$ und $0,0509 \mathrm{H}_{2} \mathrm{O}$.

4. $0,5000 \mathrm{~g}$ gaben $0,3504 \mathrm{CO}_{2}$ und $0,0443 \mathrm{H}_{2} \mathrm{O}$.

\begin{tabular}{|c|c|c|c|c|c|}
\hline \multirow{2}{*}{\multicolumn{2}{|c|}{$\begin{array}{c}\text { Berechnet für } \\
\mathrm{C}_{7} \mathrm{H}_{4} \mathrm{Br}_{\mathrm{q}} \mathrm{O}_{2} \text { : }\end{array}$}} & \multicolumn{4}{|c|}{ Gefunden: } \\
\hline & & 1. & 2. & 3. & 4 \\
\hline & 19,54 & - & - & 19,08 & 19,10 \\
\hline $\mathrm{H}$ & 0,90 & - & - & 1,1 & 0,98 \\
\hline & 72,72 & 72,18 & 72,30 & - & - \\
\hline
\end{tabular}

Tetrachlorkresorcin (Schmp. $\left.89-70^{\circ}\right) \mathrm{C}_{7} \mathrm{H}_{4} \mathrm{Cl}_{4} \mathrm{O}_{2}$. Man ubergießt 1 T. Kresorcin mit der 6 fachen Menge alkoholfreien Chloroforms und leitet unter Kühlung trockenes Chlorgas ein, (Kresorcin geht allmälich in Lơsung) bis Chlor im Ueberschuß vorhanden ist. Das Tetrachlorderivat scheidet sich teils schon von selbst in großen Krystallen aus der Lösung $a b$, der Rest wird durch Verdunstenlassen des Chloroforms erhalten. Nach dem Umkrystallisieren aus Schwefelkohlenstoff und dann aus Ligroin rhombische Tafeln, leicht loslich in Alkohol und Aether, unlosslich in Sodalösung. Ausbeute $70 \%$ der Theorie.

1. $0,3459 \mathrm{~g}$ gaben $0,4083 \mathrm{CO}_{2}$ und $0,0492 \mathrm{H}_{2} \mathrm{O}$.

2. $0,3451 \mathrm{~g}$ gaben $0,4034 \mathrm{CO}_{2}$ und $0,0349 \mathrm{H}_{2} \mathrm{O}$.

3. 0,1332 g gaben $0,2919 \mathrm{Ag} \mathrm{Cl}$.

4. $0,1242 \mathrm{~g}$ gaben $0,2709 \mathrm{Ag} \mathrm{Cl}$.

Berechnet für

$\mathrm{C}_{7} \mathrm{H}_{0} \mathrm{Cl}_{2} \mathrm{O}_{2}$ :

C 32,08

H $\quad \mathbf{1 , 5 3}$

Cl 54,16
Gefanden:

$\begin{array}{ccc}2 . & 3 . & 4 . \\ 32,15 & - & - \\ 1,14 & - & - \\ - & 54,19 & 53,94 .\end{array}$

Dichlorkresorcin (Schmp. $\left.78-79^{\circ}\right) \mathrm{C}_{7} \mathrm{H}_{8} \mathrm{Cl}_{2} \mathrm{O}_{2}$. Tetrachlorkresorcin wird mit Zinnchlorür und Salzsäure gekocht; nach dem Erkalten scheidet sich Dichlorkresorcin in feinen Nadeln sehr voluminös ab; die getrockneten Krystalle werden aus Ligroin umkrystallisiert.

1. $0,1511 \mathrm{~g}$ gaben $0,2218 \mathrm{AgCl}$.

2. $0,1422 \mathrm{~g}$ gaben $0,2093 \mathrm{AgCl}$.

3. 0,1854 g gaben $0,2938 \mathrm{CO}_{2}$ und $0,0585 \mathrm{H}_{2} \mathrm{O}$. 
Berechnet für

$\mathrm{C}_{7} \mathrm{H}_{6} \mathrm{Cl}_{9} \mathrm{O}_{2}$ :

C 43,54

H 3,11

Cl 36,85
Gefunden:

1. $2 . \quad 3$.

$\begin{array}{ccc}- & - & 43,21 \\ - & - & 3,53 \\ 36,31 & 36,39 & -\end{array}$

Monobenzoylkresorcin (Schmp. $115-116^{\circ}$ ) $\mathrm{C}_{14} \mathrm{H}_{12} \mathrm{O}_{8}$. Die Lösung von $1,2 \mathrm{~g}$ Kresorcin in $20 \%$ Sodalosung warde mit $2 \mathrm{~g}$ Benzoylchlorid geschüttelt; die dabei abgeschieden $\theta$ zähe Masse erbartete unter Wasser und wurde nach dem Trocknen über Schwefelsäure aus Ligroin umkrystallisiert; farblose Tafeln, leicht löslich in Alkohol und Aether, langsam löslich in Natronlauge.

1. $0,1793 \mathrm{~g}$ gaben $0,4822 \mathrm{CO}_{2}$ und $0,0817 \mathrm{H}_{2} \mathrm{O}$.

2. $0,1734 \mathrm{~g}$ gaben $0,4978 \mathrm{CO}_{2}$ und $0,0788 \mathrm{H}_{2} \mathrm{O}$.

Berechnet für

$$
\mathrm{C}_{14} \mathrm{H}_{18} \mathrm{O}_{8} \text { : }
$$

C 73,68

H $\quad 526$
1.

73,31

5,09
Gefunden:

2.

73,56

5,08 .

Dibenzoylkresorcin (Schmp. $\left.8^{\circ}\right) \mathrm{C}_{21} \mathrm{H}_{10} \mathrm{O}_{4}$. Kresorcin, in $15 \% \mathrm{Natronlange} \mathrm{gelöst,} \mathrm{warde} \mathrm{mit} \mathrm{therschüssigem} \mathrm{Benzoyl-}$ chlorid geschüttelt; das alkaliunlösliche unter Wasser erhärtete nnd hierauf getrocknete Reaktionsprodukt ans Petroläther umkrystallisiert; farblose Platten.

1. $0,1800 \mathrm{~g}$ gaben $0,4980 \mathrm{CO}_{2}$ and $0,0760 \mathrm{H}_{2} \mathrm{O}$.

2. $0,1830 \mathrm{~g}$ gaben $0,5078 \mathrm{CO}_{2}$ und $0,0807 \mathrm{H}_{2} \mathrm{O}$.

Berechnet für

$$
\begin{gathered}
\mathrm{C}_{21} \mathrm{H}_{16} \mathrm{O}_{\mathbf{4}} \text { : } \\
\mathrm{C} \quad 75,90
\end{gathered}
$$

H $\quad 4,85$
1.

75,14

4,72
Gefunden:<smiles>[124IH]</smiles>

\author{
2.
}

75,66

4,90 .

Diacetylkresorcin (Sdp. 293-295 $\left.{ }^{\circ}\right) \mathrm{C}_{11} \mathrm{H}_{12} \mathrm{O}_{4} . \quad 1 \mathrm{~T}$. Kresorcin wird mit 8T. Essigsäureanhydrid unter Rückfluß 1 Stunde lang gekocht; das Acetylprodukt durch Vakaumdestillation gereinigt; wasserbelle ölige Flüssigkeit, die in einem Kältegemisch nicht krystallinisch erstarrte.

1. $0,1525 \mathrm{~g}$ gaben $0,3535 \mathrm{CO}_{2}$ und $0,0839 \mathrm{H}_{2} \mathrm{O}$.

2. $0,1424 \mathrm{~g}$ gaben $0,3297 \mathrm{CO}_{2}$ und $0,0767 \mathrm{H}_{2} \mathrm{O}$.

Berechnet für

$$
\begin{array}{cc}
\mathrm{C}_{11} & \mathrm{H}_{22} \mathrm{O}_{4}: \\
\mathrm{C} & 63,46 \\
\mathrm{H} & 5,76
\end{array}
$$

Gefunden:

$$
1 .
$$$$
63,21
$$$$
6,15
$$

2.

63,14

6,02 .

Kresorcindisazobenzol (Schmp. 211-2120) $\mathrm{C}_{19} \mathrm{H}_{16} \mathrm{~N}_{4} \mathrm{O}_{2}$. Die alkoholische Lösung von $1 \mathrm{Mol}$. Kresorcin wird mit derjenigen von 21/2 Mol. Diazoamidobenzol $1 / 2$ Stunde auf dem Wasserbade digiriert, das als voluminöser roter Niederschlag abgeschiedene Azoprodnkt nach 
dem Absangen in Chloroform gelost und daraus durch Petrolather in Form scharlachroter Nadeln abgeschieden. $0,0142 \mathrm{~N}$.

1. $0,0838 \mathrm{~g}$ gaben bei $11{ }^{\circ}$ und $750 \mathrm{~mm} 12,1 \mathrm{ccm}$ Stickgas, entsprechend $0,0148 \mathrm{~N}$.

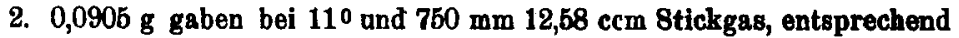

3. $0,1200 \mathrm{~g}$ gaben $0,3011 \mathrm{CO}_{2}$ und $0,0550 \mathrm{H}_{2} \mathrm{O}$.

Berechnet für $\mathrm{C}_{10} \mathrm{H}_{16} \mathrm{~N}_{4} \mathrm{O}_{9}$ :

C 68,67

H $\quad 4,81$

N 16,86
Gefunden:

$\begin{array}{ccc}1 . & 2 . & 3 . \\ - & - & 68,41 \\ - & - & 5,11 \\ 16,87 & 16,36 & -\end{array}$

Spaltung des Methylenbiskresorcin mit Natronlauge und Zinkstanb. Das bei der Spaltung entstandene Phenolgemenge, gewonnen durch Ausschtitteln des mit Soda alkalisch gemachten Filtrats der mit verdünnter Schwefelsänre neutralisierten Reaktionsflüssigkeit, ließ sich bequem durch fraktionierte Krystallisation ans heißem Benzol in Xylorcin und Kresorcin trennen. Aus der heißen Benzollösung schied sich ersteres in Form dünner farbloser Blättchen ab, die nach dem Umkrystallisieren bei $124-125^{\circ}$ schmolzen.

1. $0,1331 \mathrm{~g}$ gaben $0,3403 \mathrm{CO}_{2}$ and $0,0886 \mathrm{H}_{2} \mathrm{O}$.

2. $0,1271 \mathrm{~g}$ gaben $0,3252 \mathrm{CO}_{2}$ und $0,0832 \mathrm{H}_{\mathbf{2}} \mathrm{O}$.

Berechnet für

$$
\mathrm{C}_{7} \mathrm{H}_{10} \mathrm{O}_{2} \text { : }
$$

C 69,56

H 7,24
1.

69,71

7,44
Gefunden:

2.

69,77

$7,32$.

Dibenzoylxylorcin (Schmp. 154-1550) $\mathrm{C}_{92} \mathrm{H}_{18} \mathrm{O}_{4}$, in der üblichen Weise hergestellte farblose Prismen, unlöslich in Natronlange. $0,1555 \mathrm{~g}$ gaben $0,4340 \mathrm{CO}_{2}$ und $0,0773 \mathrm{H}_{2} \mathrm{O}$.

Berechnet für $\mathrm{C}_{92} \mathrm{H}_{18} \mathrm{O}_{4}$ :

$$
\begin{array}{ccc}
\mathrm{C} & \mathbf{7 6 , 3 0} & \mathbf{7 6 , 1 1} \\
\mathrm{H} & \mathbf{5 , 2 0} & \mathbf{5 , 5 6} .
\end{array}
$$

Gefunden:

Synthetisch (nach Pfaff) dargestelltes Xylorcin schmolz bei 124-125\%; aus Wasser krystallisierte es mit 1 Mol. $\mathrm{H}_{2} \mathrm{O}$.

1. $0,2019 \mathrm{~g}$ verloren $0,0233 \mathrm{H}_{2} \mathrm{O}$ bei $100^{\circ}$.

2. $0,2145 \mathrm{~g}$ verloren $0,0240 \mathrm{H}_{2} \mathrm{O}$ bei 1000 .

Berechnet für

$\mathrm{C}_{8} \mathrm{H}_{10} \mathrm{O}_{2}+\mathrm{H}_{2} \mathrm{O}$ :

$\mathrm{H}_{2} \mathrm{O} \quad 11,39$
Gefunden:

1.

11,54
2. $11,21$.

Das aus dem synthetisch dargestellten Xylorcin gewonnene Dibenzoat zeigte die gleichen Eigenschaften wie das oben beschriebene. (Schmp. 155\%.) 
Methylenbisx y lorcin (Schmp. $\left.251^{\circ}\right) \mathrm{C}_{17} \mathrm{H}_{20} \mathrm{O}_{4}$. $1 \mathrm{~g}$ Xylorcin, ca. $60 \mathrm{~g}$ Schwefelsäure von $24 \%$ geløst, mit $3,3 \mathrm{~g}$ Formaldehyd (kduflich) versetzt; nach 6 Stunden die abgeschiedenen Krystalle abgesaugt and aus verdïnntem Alkohol umkıystallisiert: sechseckige Tafeln, leicht 16slich in Alkohol und Aether.

1. 0,1424 g verloren bei $100^{\circ} 0,0089 \mathrm{H}_{2} \mathrm{O}$.

2. $0,1429 \mathrm{~g}$ verloren bei $100^{\circ} 0,0092 \mathrm{H}_{2} \mathrm{O}$.

Berechnet für $\mathrm{C}_{17} \mathrm{H}_{20} \mathrm{O}_{4}+\mathrm{H}_{8} \mathrm{O}$ : $\mathrm{H}_{9} \mathrm{O} \quad 5,88$
Gefunden:

$\begin{array}{cr}1 . & 2 . \\ 5,75 & 6,4 .\end{array}$

Bei der Analyse der krystallwasserfreien Substanz gaben:

1. 0,1335 g $0,3466 \mathrm{CO}_{2}$ and $0,0876 \mathrm{H}_{3} \mathrm{O}$.

2. 0,1337 g $0,3467 \mathrm{CO}_{2}$ and $0,0846 \mathrm{Hg}_{\mathrm{g}} \mathrm{O}$.

\begin{tabular}{|c|c|c|}
\hline Berechnet für & \multicolumn{2}{|c|}{ Gefunden: } \\
\hline $\begin{array}{c}\mathrm{C}_{17} \mathrm{H}_{20} \mathrm{O}_{\mathbf{8}}: \\
\mathrm{C} \quad 70,83\end{array}$ & $\begin{array}{c}1 . \\
70,79\end{array}$ & $\begin{array}{c}2 . \\
70,71\end{array}$ \\
\hline H $\quad 6,95$ & 7,26 & 7,06 \\
\hline
\end{tabular}

Monobromsylorcin (Schmp. 119-120 ) $_{8} \mathrm{C}_{9} \mathrm{BrO}_{8}$. In Chloroform verteiltes Xylorcin wird mit Brom in Chloroformlð̋sung versetzt, der nach dem Verdunsten des Lösungsmittels bleibende Rijckstand zwischen Filtrierpapier abgepreßt und nach dem Trocknen aus Petroläther umkrystallisiert: feine Nadeln.

1. $0,1621 \mathrm{~g}$ gaben $0,1398 \mathrm{AgBr}$.

2. $0,1421 \mathrm{~g}$ gaben $0,1216 \mathrm{Ag} \mathrm{Br}$.

3. 0,2189 g gaben $0,3553 \mathrm{CO}_{2}$ and $0,0792 \mathrm{H}_{9} \mathrm{O}$.

\begin{tabular}{ccccc} 
Berechnet fbr & \multicolumn{3}{c}{ Gefunden: } \\
$\mathrm{C}_{8} \mathrm{H}_{\mathbf{0}} \mathrm{Br} \mathrm{O}_{2}:$ & 1. & 2. & 3. \\
$\mathrm{C}$ & $\mathbf{4 4 , 2 4}$ & - & - & 44,26 \\
$\mathrm{H}$ & 4,15 & - & - & 4,49 \\
$\mathrm{Br}$ & $\mathbf{3 6 , 8 6}$ & $\mathbf{3 6 , 6 9}$ & $\mathbf{3 6 , 4 1}$ & -
\end{tabular}

Methylen-bis-trimethylphendiol (Schmp. 228 $)$. Aus $1 \mathrm{~g}$ des 1.2.3-Trimethylphendiol (4.6) (Schmp. 163-164\%) mittels Formaldehyd und 10prozentiger Schwefelsăure hergestellt, und durch A uáfallen der alkoholischen Løsung mit Wasser gereinigt: farblose Nadeln.

0,1074 g gaben $0,2848 \mathrm{CO}$, and $0,0724 \mathrm{H}_{\mathbf{g}} \mathrm{O}$.

Berechnet für $\mathrm{C}_{10} \mathrm{H}_{\mathbf{M}} \mathrm{O}_{\mathbf{4}}$ :

$$
\begin{array}{lr}
\text { C } & \mathbf{7 2 , 1 2} \\
\text { H } & \mathbf{7 , 5 9}
\end{array}
$$

Gefunden:

72,31

7,54 . 